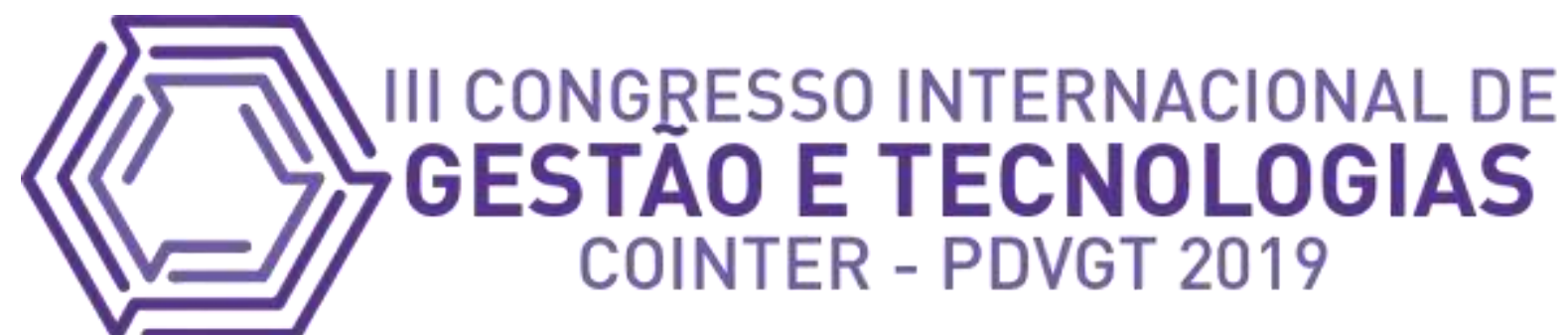

\title{
PROJETO DO SISTEMA DE TRANSMISSÃO MECÂNICA DE UM GUINCHO DE IÇAMENTO PARA LOCOMOÇÃO DE CARGAS
}

\author{
PROYECTO DEL SISTEMA DE LA TRANSMISION MECANICA DE UN GUINCHO \\ DE LEVANTAMIENTO PARA LA LOCOMOCION DE CARGAS
}

\section{DESIGN OF MECHANICAL TRASMISSION SYSTEM OF A LIFTING WINCH FOR TRANSPORTATION OF EQUIPMENTS}

\author{
Apresentação: Comunicação Oral
}

\begin{abstract}
Eduardo Ataíde de Oliveira ${ }^{1}$; Jardielson José da Costa Almeida ${ }^{2}$; Lucas Felipe de Vasconcelos ${ }^{3}$; Antonio Rodrigues F. de Carvalho ${ }^{4}$; Diógenes Linard Aquino Freitas ${ }^{5}$

DOI: https://doi.org/10.31692/2358-9728.VICOINTERPDVG.2019.0006
\end{abstract}

\begin{abstract}
Resumo
O transporte de cargas é uma atividade frequente e que exige certo esforço no meio da produção, seja para se ter a otimização do layout vigente ou para o alojamento de novos maquinários no espaço de trabalho. Para isso é requerido aparatos mecânicos que venham a facilitar a locomoção desses equipamentos de médio e grande porte, ou seja, sistemas mecânicos capazes de mover equipamentos robustos. Nesse sentido, essa produção tem por finalidade desenvolver um sistema de transmissão para um guincho, semelhante ao de uma ponte rolante, que será instalado no laboratório de práticas mecânicas do IFPE Campus Caruaru.

O projeto aborda desde a seleção do motor de indução que opera de modo satisfatório para os níveis de projeto, passando por etapas de redução por meio de elementos de máquinas flexíveis - correia e corrente - e pela caixa de engrenagens redutoras. Além disso, realizamos o dimensionamento do tipo de cabo de aço e a seleção do material da alma do mesmo, esse processo foi realizado seguindo a NBR 4309, suas recomendações e cálculos necessários. Todas as etapas da metodologia são projetadas a fim de que as especificações do projeto sejam respeitadas e válidas a níveis de segurança como carga média para içamento dos maquinários, tempo de elevação e dimensões.

O dimensionamento desses equipamentos também engloba: seleção de material dos componentes, tais como nos eixos e nas engrenagens e a análise de tempo de vida/ciclos de elementos de máquinas com nível de trabalho nominal. Engrenagens, de uma forma particular, serão projetadas baseadas na metodologia da American Gear Manufacturers Association (AGMA) que leva em consideração prioritária a resistência à flexão e ao desgaste.
\end{abstract}

\footnotetext{
${ }^{1}$ Engenharia Mecânica, Instituto Federal de Pernambuco - Campus Caruaru, eduardo_ataide@ @otmail.com

${ }^{2}$ Engenharia Mecânica, Instituto Federal de Pernambuco - Campus Caruaru, jardielsonalmeida@gmail.com

${ }^{3}$ Engenharia Mecânica, Instituto Federal de Pernambuco - Campus Caruaru, lvasconcelos1998@ gmail.com

${ }^{4}$ Engenharia Mecânica, Instituto Federal de Pernambuco - Campus Caruaru, rfc.antonio15@gmail.com

${ }^{5}$ Mestre, Instituto Federal de Pernambuco - Campus Caruaru, diogenes.freitas@ caruaru.ifpe.edu
} 
Palavras-Chave: Sistema de Transmissão, Projeto, Guincho.

\title{
Resumen
}

El transporte de cargas es una actividad frecuente y que requiere algo de esfuerzo en el medio de la produccion, sea para se ter la optimización del diseño vigente o para el alojamiento de nuevos maquinarias en el ambiente de trabajo. Para eso es solicitado aparatos mecánicos que facilitarles la locomoción de los equipamentos de medio y gran porte, o sea, sistemas mecánicos capaz de mover equipamientos fornidos. En ese sentido, esa producción tiene por finalidad desarrollar un sistema de transmisión de un guincho, semejante al de una puente rodante, que será instalado en el laboratório de las prácticas mecánicas del IFPE campus Caruaru.

El proyecto trata de la selección del motor de inducción que opera de acuerdo con los niveles del proyecto estabelecido, pasando por passos de reducción a través de los elementos de maquinas flexíbles - correa y corriente - y por la caja de engranajes reductores. Además, realizamo-nos lo dimensionamiento del tipo de cable de acero y la selección del material de la alma del mismo, ese proceso fue realizado seguiendo la NBR 4309, sus recomendaciones y cálculos necesários. Todos los passos de la metodología son deseñadas a fin de que las especificaciones del proyecto sean respetadas y validas en niveles de seguridade como la carga media para levantamento de las maquinas, tiempo de elevación y dimensiones.

El dimensionamiento de eses equipamientos tambien abarca: selección de los materiales de componentes como hachas y engranajes y la análisis de tiempo de vida/ ciclos de elementos de maquinas com nivel de trabajo nominal. Engranajes, de una forma particular, serán proyectadas según la metodología de la American Gear Manufacturers association (AGMA) que lleva en consideración prioritaria la resistencía a la flexión y al desgaste.

Palabras Clave: Sistemas de Transmisión, Proyecto, Guincho.

\begin{abstract}
Transportation of loads it's a very commom activity and demands a certain effort in the environment of production, may it be for the layout optimization or only for the lodge of a new machine in the workspace. For this task, is required tools that make it easy the transportation of those equipment's of medium and high size, this means, mechanical systems there are capable of move toughness equipment's. In this sense, this production aims the development of a transmission system for a winch, like those of an overhead crane, that will be installed in Mechanical Practices Lab at the IFPE Caruaru.

The project starts since the selection of the induction motor, whom work according to the levels of project fixed, passing through the reduction phases by means of flexible mechanical elements - belts and roller chains - and through the reduction gears. Besides that, it's was made the design of the wire rope, the selection of his material, following the NBR 4309 recommendations and calculations. Every phase of the methodology was design aiming that the specifications of project be respected and valid on the level of safety, like average load for rising of equipment, time of rising and dimensions.

The design of these mechanical elements also includes the selection of material of shafts and gears, and the analysis of the lifetime and cycles by operating at normal conditions of power. Gears, in a way, was design according to the methodology of American Gear Manufactures Association (AGMA) and considers primarily the bending fatigue failure and pitting failure.
\end{abstract}


Keywords: Transmission System, Design, Winch.

\section{INTRODUÇÃO}

O sistema de transmissão desse estudo é tomado como referência de muitos sistemas de içamento de cargas padrão do tipo ponte rolante. Composto por um tambor de cabo de aço, a caixa de engrenagens redutoras, corrente e correia, esta que se comunica com o motor elétrico e recebe sua rotação. Todos esses equipamentos estão sobre uma base de apoio que está situada na parte superior da ponte rolante. O eixo está apoiado sobre mancais. Todas as etapas do sistema de transmissão estão anotadas com sub-índices numéricos, sendo assim as grandezas tais como velocidade e forças associadas a esses estágios estão expressas da forma: 1 - motor elétrico, 2 - polia do eixo $\mathrm{AB}, 3$ - entrada do redutor, 4 - saída do redutor.

Figura 1: Componentes mecânicos do sistema de transmissão.

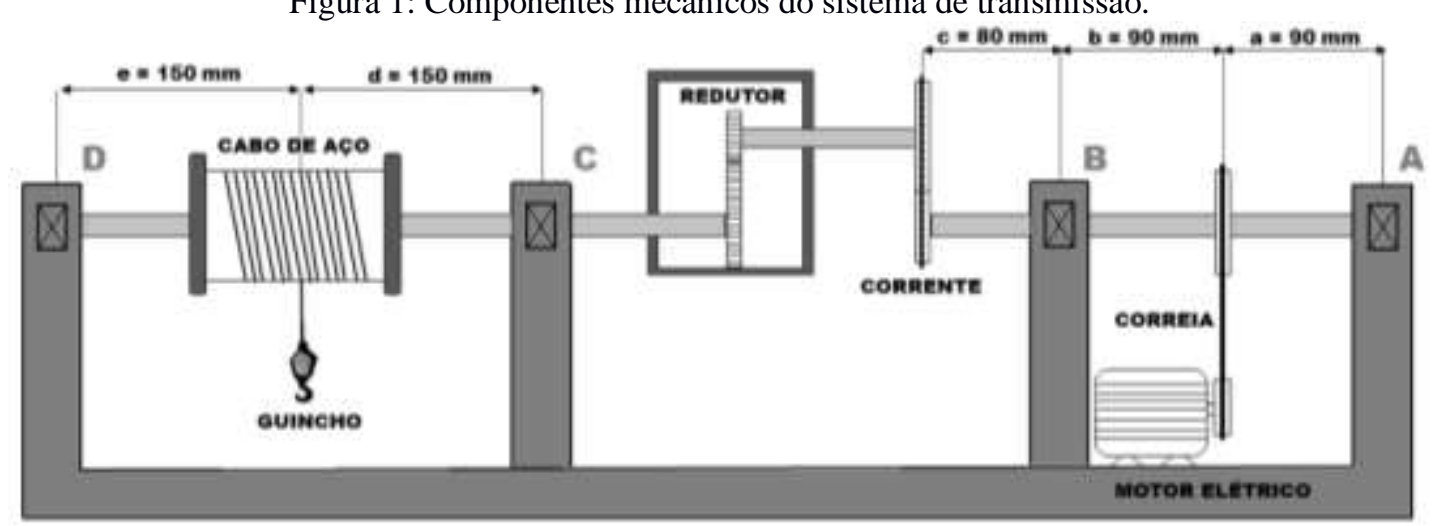

Fonte: Própria (2019)

As especificações de projeto para esse sistema foram definidas com base nas dimensões do laboratório de práticas mecânicas do IFPE Campus Caruaru, que está a ser utilizado como fonte de estudo didático para essa produção. Segue, portanto as cotas e especificações de projeto: elevar cargas de até $713 \mathrm{~kg}(7 \mathrm{kN})$ com uma velocidade de $0,3 \mathrm{~m} / \mathrm{s}$ até uma altura de 6 metros do solo. A rotação do motor elétrico que será utilizado é de $1800 \mathrm{rpm}$. As cotas $a, b, c, d$ e $e$ são distâncias tomadas dos apoios dos mancais na base de apoio do sistema de transmissão e são, respectivamente: 90 mm, 90 mm, 80 mm, $150 \mathrm{~mm}$ e $150 \mathrm{~mm}$.

\section{FUNDAMENTAÇÃO TEÓRICA}

Os sistemas de transmissão de potência - mais conhecidos por redutores ou ampliadores de velocidade - são amplamente utilizados em equipamentos mecânicos devido à necessidade de adequação de torque e velocidade fornecidos por um motor, em relação ao demandado para 
a realização de trabalho (BIANCHI, 2009). Sistemas de transmissão mecânica têm um grande campo de aplicação no âmbito da mobilidade: como setor automotivo, naval e de mecanismos industriais.

Conjuntos de transmissão são extremamente vantajosos porque são capazes de prover à carga um torque tanta vezes maior que o do motor quanto for a relação de redução (SANTOS JÚNIOR, 2002).

Nesse sentido, o correto dimensionamento dos elementos de máquinas para solicitação mecânica de um sistema como esse não somente permite um aumento do tempo de vida dos componentes, como também assegura ao projetista níveis de segurança para o equipamento. Há vários elementos de máquinas que atuam nesses sistemas, entre eles estão as correias, correntes, polias e engrenagens. De acordo com Niemann (2002), as transmissões por corrente são utilizadas para relações de multiplicação de até 6 em eixos paralelos com um rendimento da ordem de $98 \%$.

As correias, segundo Melconian (2006), possuem por característica principal a grande capacidade de multiplicação, de absorver impactos e sua perda associada, geralmente atrelada ao escorregamento e deslizamento, que representam uma perca de até 5\% de potência. Os sistemas de transmissão mecânicos encontram nas engrenagens seu principal elemento de máquina.

Pelo fato de permitirem ajustar as características da fonte de rotação de acordo com a necessidade do sistema acionado, aumentando o momento, diminuindo a velocidade de rotação e vice-versa, com o mínimo de perdas de potência (FARIA, 2009). A transmissão por engrenagens apresenta um elevado rendimento, comparativamente com outros sistemas de transmissão mecânica.

\section{METODOLOGIA}

Partindo-se da velocidade angular do eixo do tambor (seção CD), tem-se ideia da redução global que o sistema necessita, é, portanto, feita uma ponderação do quanto será o fator de redução em cada um dos três elementos (correia, corrente e engrenagens). Uma vez estabelecido, é feito o estudo particular do componente. O organograma ilustra o processo de 
desenvolvimento do projeto.

Figura 2: Organograma das fases do projeto do dimensionamento.

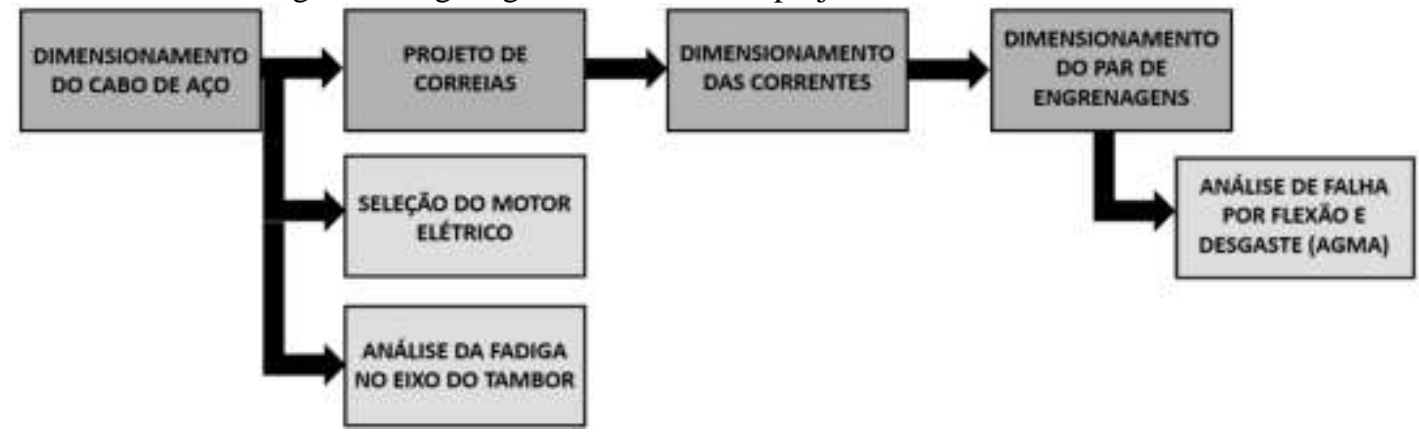

Fonte: Própria (2019)

\section{1 - Dimensionamento do cabo de aço e tambor}

O estudo do componente cabo de aço consiste em encontrar as cargas de trabalho a partir de um fator de segurança recomendado, segundo a NBR 4309, a recomendação de coeficiente de segurança para um cabo de aço de elevação de aplicação em pontes rolantes varia de 6 a 8 . O coeficiente de segurança é a razão da Carga de Ruptura Mínima CMR e a Carga de Trabalho CT, como explicita a equação (1):

$$
n=\frac{C M R}{C T}
$$

A Carga de Trabalho leva em consideração forças de atrito e inércia, $W$ é a carga a ser elevada em $N, m$ o número de cabos de aço para a operação $(m=1)$, $\omega$ é o peso por metro do cabo de aço e $l$ é o comprimento suspenso do cabo (altura de elevação) que será de 6 metros. Da tabela 17-24 do Shigley (2008), temos que para içamento padrão, o cabo utilizado é o $6 \times 19$ que apresenta um $\omega=36,18 \times 10^{-3} \mathrm{~N} / \mathrm{m}$, $a$ é referente a aceleração que será de 0,015 $\mathrm{m} / \mathrm{s}^{2}$ e $g=9,81 \mathrm{~m} / \mathrm{s}^{2}$.

Pelo fato de o diâmetro do cabo ser um fator limitante, visto que não foi selecionado previamente, tomaremos, portanto, uma média entre os valores do intervalo dado dos tamanhos padronizados da tabela 17-24 (Shigley, 2008). Obtendo $d=38 \mathrm{~mm}$. Aplicando esses valores 
na equação (2), temos:

$$
\begin{gathered}
C T=\left(\frac{W}{m}+\omega l\right)\left(1+\frac{a}{g}\right) \\
C T \approx 7325 N
\end{gathered}
$$

Tomando o coeficiente de segurança máximo, 8, temos: $C M R=58,6 \mathrm{kN}$

O manual técnico de Cabos de Aço (CIMAF, 2012) o qual essa produção tomou como base para estudo desse elemento, fornece os valores de CMR em tf, sendo assim $C M R=$ 5,977 tf de um $6 x 19$ alma de aço, o diâmetro da bitola do cabo $(d=9,5 \mathrm{~mm})$. Com isso, sabe-se que o diâmetro $\left(D_{\text {tambor }}\right)$ para o tambor se situa numa faixa de 30 a 45 vezes o diâmetro do cabo, pondo uma margem de segurança, o diâmetro do tambor escolhido será da ordem de 37 vezes o diâmetro do cabo. $D_{\text {tambor }}=351,5 \mathrm{~mm}$.

Tendo em mãos o diâmetro do tambor e a velocidade de elevação da carga, determinamos a rotação do eixo pela expressão (3):

$$
V=\omega \times r
$$

Resultando assim, $\omega_{4}=16,3 \mathrm{rad} / \mathrm{s}$. A potência desenvolvida no eixo é tida pela expressão (4):

$$
H_{4}=\left(r_{\text {tambor }} \times C T\right) \times \omega_{4}
$$

Resultando em 2,975 cv, porém, ao considerar perdas nos estágios de transmissão, a potência que o motor elétrico terá de desenvolver será maior. Estimando uma perda de potência nos estágios de redução da correia, corrente e engrenagens de 5, 3 e $0 \%$, respectivamente. Temos que a potência do motor deverá ser de:

$$
H_{\text {motor }}=\frac{H_{4}}{(1 \times 0,97 \times 0,95)}=\frac{2,9755}{0,9215} \cong 3,5 \mathrm{cv}
$$

Por questões de disponibilidade comercial e superdimensionamento de segurança para a aplicação, será selecionado um motor de potência 4,0 cv. Segue abaixo na tabela 1 algumas 
de suas especificações:

Tabela 1: Especificações técnicas do motor selecionado

\begin{tabular}{|l|l|}
\hline \multicolumn{2}{|c|}{ Especificações técnicas } \\
\hline Rotação nominal & $1800 \mathrm{rpm}$ \\
\hline Tensão & $220 / 380 \mathrm{~V}$ \\
\hline Número de polos & 4 \\
\hline Frequência & $60 \mathrm{~Hz}$ \\
\hline Fonte: Própria (2019)
\end{tabular}

\section{2 - Dimensionamento do eixo do tambor por critério de falha}

Para realizar a análise de fadiga, é necessário determinar o torque médio $\left(T_{m}\right)$ e o momento alternado $\left(M_{a}\right)$. As equações de equilíbrio por meio do diagrama de corpo livre (figura 3) fornecem que as reações nos mancais $\mathrm{C}$ e $\mathrm{D}$ : com as reações de ambos os pontos em $\mathrm{z}$ sendo nulas, $C_{y}=-3459,645 \mathrm{~N}$ e $D_{y}=3519,645 \mathrm{~N}$.

Figura 3: Diagrama do corpo livre para o eixo CD

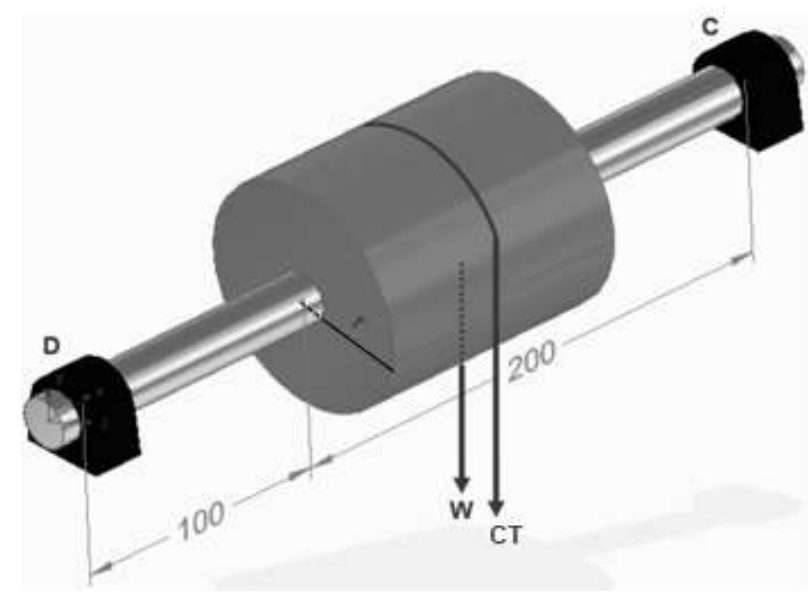

Fonte: Própria (2019)

O diagrama de Momento Fletor nos fornece o maior momento aplicado no eixo e resulta em $M_{a}=527,94$ N. $m$ e $T_{m}$ é obtido pelo produto $r \times C T, T_{m}=1230,25 \mathrm{~N}$. m. Para um eixo rotacionando com flexão e torção constantes, ou seja, a tensão de flexão é completamente reversa e a torção estável, $\log M_{m}=T_{a}=0$. Pelo critério de falha de DE-Goodman, 
estimando um fator de segurança $n=2$. Teremos por (5):

$$
\begin{gathered}
d=\left(\frac{16 n}{\pi}\left\{\frac{1}{s e}\left[4\left(K_{f} M_{a}\right)^{2}\right]^{\frac{1}{2}}+\frac{1}{s_{u t}}\left[3\left(K_{f s} T_{m}\right)^{2}\right]^{\frac{1}{2}}\right\}\right)^{\frac{1}{3}} \\
d_{C D}=53,85 \mathrm{~mm}
\end{gathered}
$$

Alguns fatores e grandezas estão apresentadas na tabela 2:

Tabela 2: Fatores e Tensões da análise de falha por fadiga do eixo CD

\begin{tabular}{|c|c|c|c|}
\hline $\begin{array}{c}\text { Limite de resistência }\left(\mathrm{S}_{\mathrm{ut}}\right) \mathrm{p} / \\
\mathrm{SAE} 1035-\mathrm{HR}:\end{array}$ & $500 \mathrm{MPa}$ & Limite de Endurança $\left(\mathrm{S}_{\mathrm{e}}\right):$ & $\begin{array}{c}149,625 \\
\mathrm{MPa}\end{array}$ \\
\hline Fator de superfície $\left(\mathrm{k}_{\mathrm{a}}\right):$ & 0,665 & $\begin{array}{c}\text { Fator concentrador de tensão } \\
\text { flexional }\left(\mathrm{K}_{\mathrm{f}}\right):\end{array}$ & 1,49 \\
\hline Fator de tamanho $\left(\mathrm{k}_{\mathrm{b}}\right):$ & 0,9 & $\begin{array}{c}\text { Fator concentrador de tensão } \\
\text { torcional }\left(\mathrm{K}_{\mathrm{fs}}\right):\end{array}$ & 1,13 \\
\hline
\end{tabular}

Fonte: Própria (2019)

\section{3 - Reduções do sistema}

É tomado como fator limitante de projeto que as taxas de redução devam ser menores que 10, para não super-requerer aspectos mecânicos dos equipamentos. A figura 4 mostra esquematicamente a redução por estágios relacionado a cada elemento de máquina:

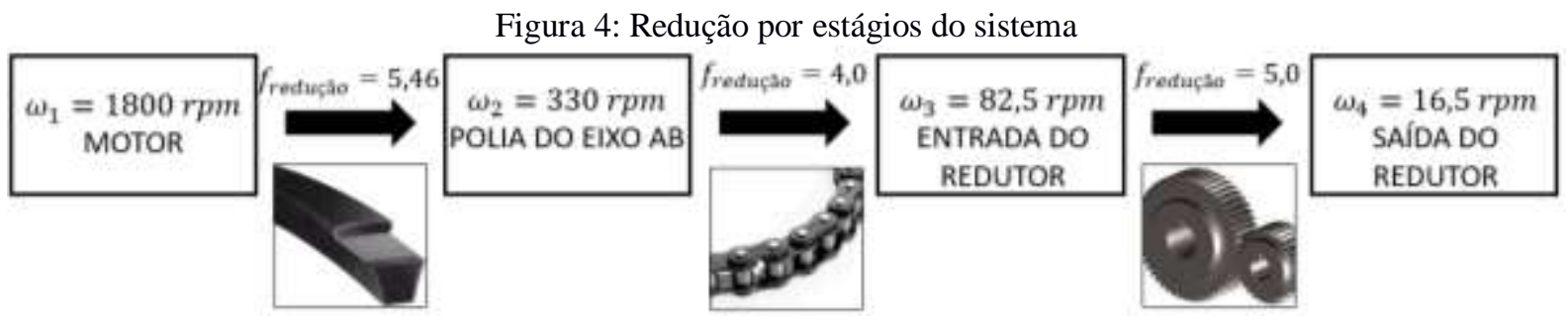

Fonte: Própria (2019)

\section{4 - Dimensionamento e análise de vida: correia}

Na correia, há uma perda de 5\% na transmissão da potência nominal do motor, então a potência nominal usada na definição de demais parâmetros deve levar em conta tal perda, assim, sendo a potência desenvolvida na saída do motor $4,0 \mathrm{cv}$ (aproximadamente $3 \mathrm{~kW}$ ): $H^{\prime}{ }_{\text {nom }}=$ $0,95 \times H_{\text {nom }}=2,85 \mathrm{~kW}$.

A tabela 17-12 do Shigley (2008) relaciona as potências para diferentes velocidades e diferentes diâmetros mínimos, com base nessa, o diâmetro da polia motora: $d_{p_{1}}=155 \mathrm{~mm}$. Com a redução estabelecida de 5,46, tem-se: $d_{p_{2}}=5,46 \times 155=846,30 \mathrm{~mm}$. 
A velocidade periférica é tida a partir do diâmetro da polia motora, pela expressão (6), onde $\mathrm{n}$ é a rotação de saída do motor em rps:

$$
V_{\text {per }}=\pi d_{p_{1}} n
$$

A velocidade periférica de $14,6 \mathrm{~m} / \mathrm{s}$ situa-se numa faixa de operação boa, visto que velocidades acima de $25 \mathrm{~m} / \mathrm{s}$ ou abaixo de $5 \mathrm{~m} / \mathrm{s}$ ocasionam problemas no projeto. Interpolando valores de velocidade periférica dado o diâmetro primitivo da roldana e o tipo de correia (tipo B) na tabela 17-12 do Shigley (2008), temos que a potência tabelada $\left(H_{t a b}\right)$ é de $2,89 \mathrm{~kW}$.

Da tabela 17-10, define-se o comprimento da circunferência interna e da tabela 17-11 do Shigley (2008), o comprimento a ser adicionado. São, respectivamente: $L_{c}=4500 \mathrm{~mm}$ e $L_{a d}=45 \mathrm{~mm}$. Temos assim um comprimento primitivo $\left(L_{p}\right)$ de $4545 \mathrm{~mm}$.

Da equação (7), define-se a distância entre centros C:

$$
C=0,25\left\{\left[L_{p}-\frac{\pi}{2}\left(d_{p_{2}}+d_{p_{1}}\right)\right]+\sqrt{\left[L_{p}-\frac{\pi}{2}\left(d_{p_{2}}+d_{p_{1}}\right)\right]^{2}-2\left(d_{p_{2}}-d_{p_{1}}\right)^{2}}\right\}
$$

Resultando $C=1445 \mathrm{~mm}$. Esse dimensionamento respeita a desigualdade $d_{p_{2}}<C<$ $3\left(d_{p_{2}}+d_{p_{1}}\right)$, portanto será mantida no projeto.

Determinar os fatores de correção de abraçamento $\left(k_{1}\right)$ e de comprimento $\left(k_{2}\right)$ nos permite obter a potência admissível. Para isso é necessário encontrar o ângulo de abraçamento através da equação (8):

$$
\varphi=\pi-2 \operatorname{sen}^{-1}\left(\frac{d_{p_{2}}-d_{p_{1}}}{2 C}\right)
$$

Com os devidos valores previamente obtidos, tem-se que o ângulo de abraçamento $(\varphi)$ resulta em 2,66 rad, ou 152,4 ${ }^{\circ}$. Esse valor nos possibilita fazer uso da tabela 17-13 do Shigley (2008) que relaciona diferentes fatores $k_{1}$ para correias planas em V, e ângulos $\varphi$. Por meio da interpolação na tabela 3: 
Tabela 3: Tabela para interpolação do fator $\mathrm{k}$

\begin{tabular}{|c|c|}
\hline$\varphi$ (graus) $^{\circ}$ & $k$ \\
\hline $156,9^{\circ}$ & 0,80 \\
\hline $152,4^{\circ}$ & $\mathrm{k}$ \\
\hline 151 & 0,81 \\
\hline
\end{tabular}

Fonte: Shigley (2008). Adaptado pelos autores

Com isso, $k_{1}=0,808$. O fator de correção de abraçamento $\left(k_{2}\right)$ é obtido na tabela 1714, que relaciona o tipo de correia (tipo B) para um determinado comprimento primitivo. Logo, $k_{2}=1,15$. A potência admissível, é, portanto, encontrada pela equação (9):

$$
H_{a d m}=k_{1} k_{2} H_{t a b}
$$

Com os devidos valores, tem-se que $H_{a d m}=2,68 \mathrm{~kW}$. Os valores de potência admissível e potência de desenho são importantes pois a partir de uma correlação, irá se determinar o número de correias para o projeto. A expressão (10) utiliza a potência nominal $\left(H_{\text {nom }}\right)$, um fator de serviço $\left(k_{s}\right)$ e um fator de desenho $\left(n_{d}\right)$ para encontrar $H_{d}$.

$$
H_{d}=H_{n o m} k_{s} n_{d}
$$

Adotando um fator de desenho unitário e um fator de serviço 1,3 para choque médio, temos que $H_{d}=3,705 \mathrm{~kW}$. O número de correias $\left(N_{b}\right)$ deve ser maior ou igual à razão da potência de desenho pela admissível:

$$
N_{b} \geq \frac{3,705}{2,68} \rightarrow N_{b} \geq 1,38
$$

Serão necessárias duas correias para essa transmissão $N_{b}=2$. Através dessas informações, temos que o fator de segurança $\left(n_{f s}\right)$ é expresso através de $(11)$ e se relaciona com $H_{a d m}, N_{b}, H_{n o m}$ e $k_{s}$ :

$$
n_{f s}=\frac{H_{a d m} N_{b}}{H_{n o m} k_{s}}
$$


O fator de segurança é de 1,45. Para a determinação das forças envolvidas foi feito uma rotina de procedimentos com auxílio do Excel ${ }^{\circledR}$, a fim de ser eficiente e sumarizar cálculos robustos para o cálculo de vida da correia.

Tabela 4: Procedimentos para cálculo de tempo de vida das correias

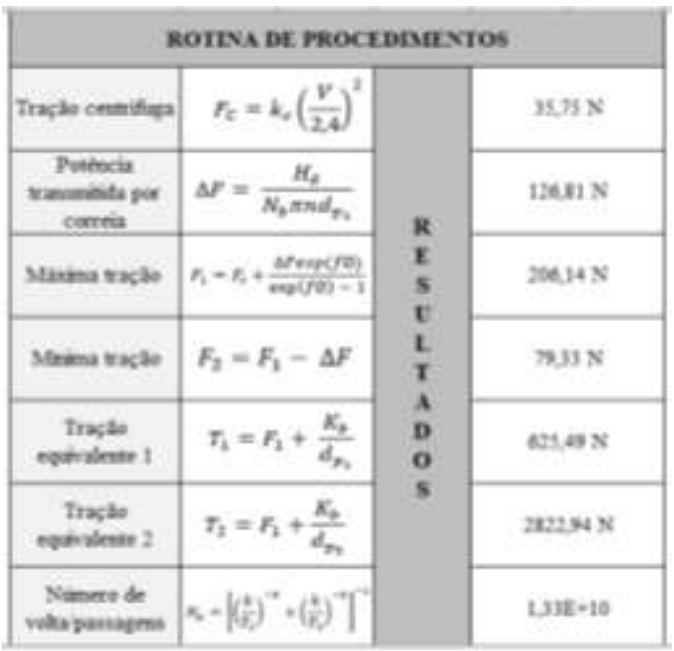

Fonte: Própria (2019)

Sendo assim, o número de voltas $1,327 \times 10^{10}$ passagens. Podemos agora calcular o tempo de vida através de (12):

$$
t=\frac{N_{p} L_{p}}{3600 V}
$$

O tempo de vida é da grandeza de 87000 horas.

\section{5 - Dimensionamento da corrente}

Levaremos em consideração a capacidade de carga da corrente fazendo suas devidas correções, primeiramente, considerando a perda de $3 \%$ de potência como foi citado na seção de seleção do motor, temos que a potência é então de 3,88 cv. Uma correção é feita a partir do fator de serviço $\left(k_{S}\right)$, para condições moderadas de operação e motor elétrico, temos que o fator de serviço é 1,3, sendo assim, por (13):

$$
H_{\text {proj }}=k_{s} H
$$

A potência de projeto é de 5,04 cv. A tabela 17-20 do Shigley (2008) nos fornece a capacidade em cavalo-vapor para várias velocidades da roda dentada. Foi necessário fazer uma interpolação para uma velocidade de $330 \mathrm{rev} / \mathrm{min}$ (velocidade da polia do eixo $\mathrm{AB}$ ). 
Tabela 5: Capacidade em cavalos para corrente de filetes de fila única

\begin{tabular}{|c|c|c|c|c|c|c|c|c|c|c|c|c|c|c|}
\hline & \multicolumn{10}{|c|}{ Número de corrente AN51 (p/ roda dentada de 17 dentes) } \\
\hline revimin & 25 & 35 & 40 & 41 & 50 & 60 & 80 & 100 & 120 & 140 & 160 & 180 & 200 & 240 \\
\hline 300 & 0,23 & 0,78 & 1,85 & 1,02 & 3,61 & 6,2 & 14,5 & 27,7 & 46,8 & 72,4 & 105 & 145 & 198 & 310 \\
\hline 330 & 0,251 & 0,349 & 2,015 & 1,11 & 3,928 & 5,749 & 15,76 & 30,16 & 50,94 & 78,82 & 114,3 & 157,9 & 209,8 & 324,7 \\
\hline 400 & 0,3 & 1,01 & 2,4 & 1,32 & 4,67 & 8,03 & 18,7 & 35,9 & 60,6 & 93,8 & 136 & 183 & 249 & 359 \\
\hline
\end{tabular}

Fonte: Shigley (2008). Adaptado pelos autores

Onde cada cor indica o tipo de lubrificação: tipo A (azul) - por gotejamento ou manual; B (verde) - por disco ou por banho; tipo C (amarelo) - por corrente de óleo; tipo C' (vermelho) - por corrente de óleo com esfolamento. O fator de correção do número de dentes $\left(k_{1}\right)$ da tabela 17-20 Shigley (2008) para uma corrente de filetes únicos e roda dentada de 14 dentes é 0,78. Outro fator de correção se faz necessário para corrigir a potência transmitida, o fator $k_{2}$ relativo ao número de fileiras, da tabela 17-23 do Shigley (2008) não é necessário pois trata-se de uma fileira única. Pela equação (14):

$$
H_{\text {corr }}=k_{1} k_{2} H_{\text {proj }}
$$

A potência corrigida é de 3,9312 cv. Segue abaixo os resultados na tabela 6:

Tabela 6: Relação dos tipos de correia para diferentes configurações

\begin{tabular}{|c|c|c|c|c|c|c|c|c|c|c|c|c|c|c|}
\hline & 25 & 35 & 40 & 41 & 50 & 60 & 80 & 100 & 120 & 140 & 150 & 180 & 200 & 240 \\
\hline pies & 0,19578 & 0,6622 & 1,5717 & 0,8658 & 3,06384 & 5,2642 & 12,2928 & 23,5248 & 39,73 & 61,4796 & 89,154 & 123,162 & 163,644 & 253,266 \\
\hline Dupla & 32826 & 1,125774 & 7189 & 1,47186 & 5,208528 & 8,949174 & 20,89776 & 39,99216 & 57,54644 & 104,5153 & 151,5618 & 209,3754 & 278,1948 & 430,5522 \\
\hline & & & & & & 13,16055 & & & & & & & 11 & 633,165 \\
\hline Quadru & 0,646074 & 2,185326 & 18661 & 85714 & 10,11057 & 17,37193 & 40,56624 & 77,63184 & 131,1196 & 202,8827 & 294,2082 & 406,4346 & 540,0252 & 835,7778 \\
\hline
\end{tabular}

Fonte: Própria (2019)

Como a capacidade da corrente de $\mathrm{n}^{\circ} 60$, na configuração simples, foi a mais próxima e ligeiramente maior do que a $H_{\text {projetado }}$ escolheremos ela para aplicarmos ao nosso sistema.

Da tabela 17-9, poderemos determinar alguns parâmetros da corrente para calcularmos limites de potência e verificarmos se a corrente está dentro desses limites. O limite de potência baseada na fadiga da placa de ligação é dado pela equação (15):

$$
H_{\text {lim }, p l}=0,004 \times N_{1}^{1,08} \times n_{1}^{0,9} \times p^{(3-0,07 p)}
$$

Onde: $N_{1}$ é o número de dentes da roda dentada menor (14), $n_{1}$ é a velocidade angular 
(330 rev/min), e p é o passo (0,75 in). Assim, $H_{l i m, p l}=5,5 \mathrm{cv}$. Fazendo o uso da equação (16), para o limite de potência baseado no rolete e na bucha e da equação (17), para o limite de potência baseado no desgaste excessivo.

$$
\begin{gathered}
H_{\text {lim }, R B}=\frac{1000 \times K r \times N_{1}^{1,5} \times p^{0,8}}{n_{1}^{1,5}} \\
H_{\text {lim }, D C}=\left(\frac{N_{1} \times n_{1} \times p}{110,84}\right) \times\left(4,413-2,073 \times p-0,0274 \times N_{2}\right)-\left(\frac{\ln n_{2}}{1000}\right) \\
\times(1,59 \times \log p+1,873)
\end{gathered}
$$

$118 c v$ e 41,4 cv, respectivamente. Como a potência projetada não ultrapassa esses níveis, poderemos utilizar essa corrente no nosso sistema. Com isso, podemos determinar os aspectos geométricos e dimensionais da corrente que seguem apresentados na tabela 7 .

Tabela 7: Especificações da corrente

\begin{tabular}{|l|l|}
\hline Diâmetro do pinhão $\left(D_{P}\right):$ & 3,37 in \\
\hline Diâmetro da coroa $\left(D_{c}\right):$ & 13,37 in \\
\hline Número de passos $(\mathrm{L}):$ & 96 passos \\
\hline
\end{tabular}

Fonte: Própria (2019)

\section{6 - Dimensionamento das engrenagens redutoras}

Para o dimensionamento do par de engrenagens, temos que o fator de redução é de 5, logo, por (18). Os sub-índices 3 e 4 são relativos ao pinhão e coroa, respectivamente.

$$
r_{4}=5 \times r_{3}
$$

Iniciando com uma análise de interferência para engrenagens cilíndricas de dentes retos, temos que o número mínimo de dentes no pinhão é dado por (19):

$$
N_{3}=\frac{2 k}{\left(1+2 m_{G}\right) \sin ^{2} \emptyset}\left(m_{G}+\sqrt{m_{G}^{2}+\left(1+2 m_{G}\right) \sin ^{2} \emptyset}\right)
$$

Para uma razão de engrenamento (fator de redução) igual a 5 e tomando o ângulo de pressão como $20^{\circ}$ e que $\mathrm{k}=1$, pois temos que os dentes possuem altura completa. $\mathrm{O}$ menor número de dentes do pinhão para que não ocorra interferência é 16. Para a coroa, o maior 
número de dentes da engrenagem é dado pela equação (20):

$$
N_{4}=\frac{N_{3}^{2} \sin ^{2} \emptyset-4}{4-2 N_{3} \sin ^{2} \varnothing}
$$

A expressão nos dá como resultado 100 dentes, logo, dado o intervalo obtido, foi projetado um pinhão com 18 dentes, e uma coroa com 90. Tomando como fator de projeto um módulo (m) de 6mm, temos, através dos devidos cálculos:

Tabela 8: Dimensões do par engrenado

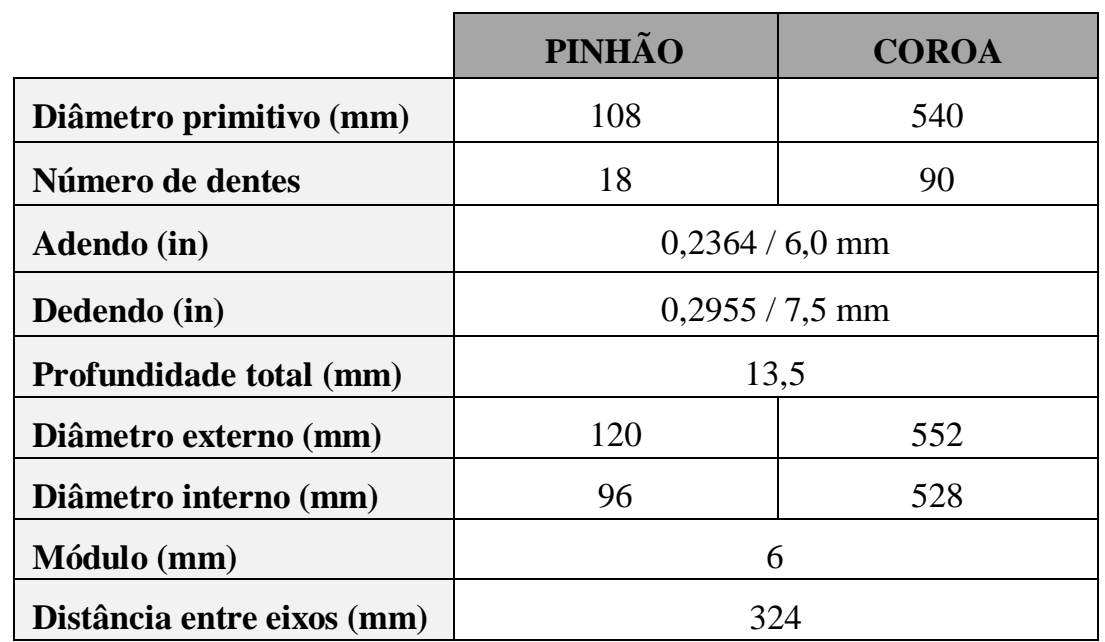

Fonte: Própria (2019)

\section{7 - Análise de falha de flexão e desgaste nas engrenagens}

Uma vez projetado os aspectos dimensionais, a análise das forças é imprescindível para o projeto contra falha de desgaste e flexão. Calculando a carga transmitida no pinhão por (21):

$$
W_{t}=F_{34}^{t}=\frac{60000 H_{3}}{\pi d_{3} n_{3}}
$$

Sendo $\mathrm{H}$ a potência que chega no redutor passando pelos estágios de redução de correia e corrente, temos que: $H_{3}=H_{\text {motor }} \times$ Perdas $=4 \times 0,97 \times 0,92=3,57 \mathrm{cv}=2625,731 \mathrm{~W}$

Com $d_{3}=108 \mathrm{~mm}$ e a celeridade $n_{3}=\omega_{3}=82,5 \mathrm{rev} / \mathrm{min}$. Portanto: A carga transmitida é de 5,628 $\mathrm{kN}$. Sabendo que o ângulo de contato $\phi$ é $20^{\circ}$, podemos determinar a componente radial e o módulo da força. A figura 5 abaixo mostra um diagrama de corpo livre das forças aplicadas no par de engrenamento. 


$$
F_{34}^{r}=2,048 k N ; \quad F_{34}=5,989 k N
$$

Figura 5: Diagrama do corpo livre das forças aplicadas nas engrenagens

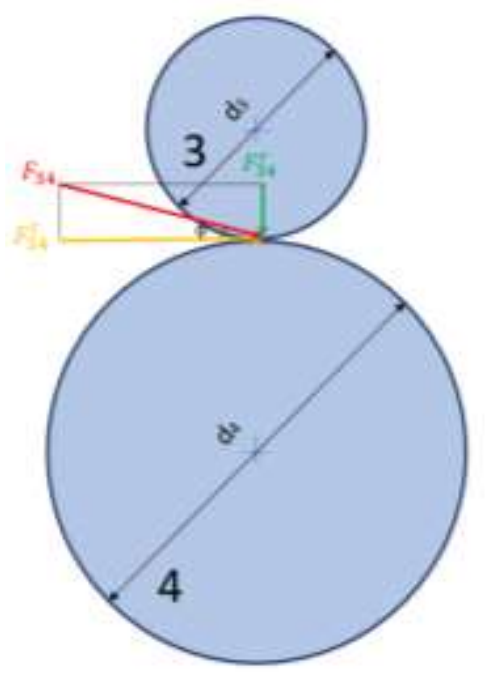

Fonte: Própria (2019)

Realizando a análise da velocidade na linha primitiva, e sabendo que $d$ é dado em polegadas e $n$ em rpm, temos por (22):

$$
V=\frac{\pi d_{3} n_{3}}{12}
$$

A análise de falha de desgaste e flexão da metodologia da AGMA é feita em unidades inglesas, portanto, a fim de se adaptar a essas unidades temos que a carga transmitida será $W^{t}=$ 1265,25 lbf. As equações de tensão da AGMA, considerando todos os fatores, são duas a flexional (23):

$$
\sigma_{f}=W^{t} K_{o} K_{v} K_{s} \frac{P_{d}}{F} \frac{K_{m} K_{B}}{J}
$$

E a de resistência ao crateramento (24):

$$
\sigma_{c}=C_{p} \sqrt{W^{t} K_{o} K_{v} K_{s} \frac{K_{m}}{d_{p} F} \frac{C_{f}}{I}}
$$

As equações do coeficiente de segurança AGMA $S_{F}$ para flexão e contato $S_{H}$ são expostas a seguir por (25) e (26), respectivamente:

$$
\begin{gathered}
S_{F}=\frac{S_{t}}{\sigma_{f, a d m}} \frac{Y_{N}}{K_{T} K_{R}} \\
S_{H}=\frac{S_{c}}{\sigma_{c, a d m}} \frac{Z_{N} C_{H}}{K_{T} K_{R}}
\end{gathered}
$$

Serão feitas, portanto, quatro análises. Flexão e Contato para o Pinhão e Flexão e 
Contato para a Coroa. Para determinar os fatores e coeficientes, serão feitas considerações de projeto baseadas na seleção do material e suas propriedades.

O pinhão é de Aço grau 1 com dureza de superfície e do dente de 240 HB (módulo de elasticidade $30 \mathrm{Mpsi}$ ), a coroa é de Aço grau 1 e possui dureza de superfície e do dente de 200 HB (módulo de elasticidade $2300 \mathrm{Mpsi}$ ). O pinhão irá rotacionar por $10^{8}$ ciclos e a coroa por $2 \times 10^{7}$ ciclos. Largura da face do par engrenado 2,97 in, possuem dentes não coroados e por consideração de projeto os mancais estão imediatamente adjacentes ao redutor e as engrenagens do redutor estão confinadas.

O redutor também é comercialmente disponível, por fim, consideraremos que o projeto possui confiabilidade de 99\%. Aplicando as devidas relações, analisando figuras, tabelas e tomando liberdade de escolha por justificativa de projetos para alguns quesitos dos quais os fatores irão depender, tem-se todos os resultados tabelados abaixo (9):

Tabela 9: Fatores e coeficientes do projeto

\begin{tabular}{|c|c|c|c|}
\hline \multicolumn{4}{|c|}{ Fatores e cocficicutes de projeto } \\
\hline $\mathbf{K}_{0}$ & 1,00 & $\mathbf{J}_{3}$ & 0.320 \\
\hline $\mathbf{K V}$ & 1.130 & $\mathbf{J}_{4}$ & 0.420 \\
\hline $\mathbf{K} \times, 3$ & 1,135 & $\mathrm{C}_{\mathrm{p}}$ & 2300 vpsi \\
\hline$K_{3,4}$ & 1.144 & $\mathrm{Cr}$ & 1.00 \\
\hline Pd & 4.23 dentes $/$ in & dp3 & 4,252 in \\
\hline $\mathbf{F}$ & 2.97 in & I & 0.134 \\
\hline $\mathrm{Km}$ & 1,129 & $\mathbf{K} \mathbf{T}$ & 1.00 \\
\hline $\mathrm{KB}, 3$ & 1,00 & KR & 1,00 \\
\hline $\mathbf{K}_{\mathbf{B}, 4}$ & 1,00 & $\mathbf{Z N}, 3_{3}$ & 0.948 \\
\hline $\mathrm{Si}, 3$ & $31.352 \mathrm{kpsi}$ & $\mathbf{Z X}, 4$ & 0.984 \\
\hline St,4 & $28,26 \mathrm{kspi}$ & $\mathbf{Y} \times 3$ & 0,977 \\
\hline $\mathrm{Se}, 3$ & $106.28 \mathrm{kpsi}$ & $\mathbf{Y N}_{4}$ & 1,005 \\
\hline $\mathrm{S}_{\mathrm{c}, 4}$ & $93,5 \mathrm{kpsi}$ & & \\
\hline Cus 3 & 1,00 & & \\
\hline Сн.4 & 1.01 & & \\
\hline
\end{tabular}

Fonte: Própria (2019)

Calculando a falha por flexão no pinhão e na coroa por (23):

$$
\left(\sigma_{f, a d m}\right)_{3}=8157,82 p s i ;\left(\sigma_{f, a d m}\right)_{4}=6267 p s i
$$

E consequentemente o coeficiente de segurança por (25):

$$
\left(S_{F}\right)_{3}=3,75 ;\left(S_{F}\right)_{4}=4,53
$$

Calculando a falha por desgaste no pinhão e na coroa por (24):

$$
\left(\sigma_{c, a d m}\right)_{3}=75724 p s i ;\left(\sigma_{c, a d m}\right)_{4}=76037 p s i
$$

E, da mesma forma, obtendo os coeficientes de segurança por (26):

$$
\left(S_{H}\right)_{3}=1,33 ;\left(S_{H}\right)_{4}=1,178
$$




\section{RESULTADOS E DISCUSSÃO}

Para o dimensionamento do cabo de aço para içamento de cargas em uma ponte rolante utilizamos um fator de segurança de 8, a carga de trabalho calculada segundo a NBR 4309 e com o valor encontrado juntamente com o fator de segurança conseguimos encontrar a carga de ruptura mínima em toneladas força de 5,977 tf , o diâmetro de tambor encontrado foi de $351,5 \mathrm{~mm}$ e a potência calculada do motor foi de 3,5 cv, mas selecionamos um motor de potência maior como uma forma de garantir que a potência requerida seja sempre atendida, mesmo que o sistema apresente alguma mudança. Realizamos os cálculos de momento fletor e torque máximo e encontramos os valores respectivamente de $M_{a}=527,94 \mathrm{~N}$. $\mathrm{m}$ e $T_{m}=$ 1230,25 N.m.

Utilizamos o critério de DE-Goodman para análise de fadiga no eixo do tambor. O sistema possui 3 reduções entre quatro estágios, ou seja, desde o motor elétrico com velocidade angular $\omega_{1}=1800 \mathrm{rpm}$ no primeiro estágio, até a saída do redutor com velocidade $\omega_{4}=$ 16,5 rpm no último estágio.

O dimensionamento das correias foi realizado com base na redução prevista em projeto de 5,46, dimensiona-se uma polia motora de $155 \mathrm{~mm}$ de diâmetro e com essas duas informações encontramos o tamanho da polia movida de $846,3 \mathrm{~mm}$. O comprimento da circunferência interna foi de 4500 com um adicional de $45 \mathrm{~mm}$ devido ao tipo da correia (no caso, tipo B) e a distância entre os centros foi estabelecida para $\mathrm{C}=1445 \mathrm{~mm}$. Por fim o número de correias dimensionadas para o projeto foi de 2 correias, fator de segurança obtido foi de 1,45 e o tempo de vida em horas foi de 87000 horas.

Para a corrente a redução estabelecida foi de 4, calculamos a potência de projeto e a partir dela corrigimos seu valor para uma capacidade de corrente de número 60 e configuração simples, assim encontramos uma potência e sua configuração acima da projetada. Uma análise quanto a limites de potência baseada em placa de ligação, no rolete e na bucha e ainda no desgaste excessivo se fez necessário e os valores encontrados são respectivamente: $H_{l i m, p l}=$ $5,5 \mathrm{cv} ; H_{l i m, R B}=118 \mathrm{cv}$ e $H_{\text {limp }, D C}=41,4 \mathrm{cv}$.

Por fim, as engrenagens foram dimensionadas para uma redução de 5. A potência transmitida foi de 2,6257 $\mathrm{kW}$ e a força transmitida $W^{t}=1265,25 \mathrm{lbf}$. Quatro análises de flexão e desgaste foram realizadas considerando pinhão e coroa. Os resultados encontrados do projeto foram expostos na tabela (10) mostrando as principais informações encontradas para cada componente. 
Ao se realizar um comparativo entre os coeficientes de segurança de flexão e contato, devemos ter cuidado para analisar os termos. Visto que, o coeficiente de segurança de resistência ao contato está elevado a potência de um meio e, portanto, nossa analise não está sendo feita com as mesmas proporções comparativas. Para isso:

$$
\left(S_{H}\right)_{3}^{2} e\left(S_{H}\right)_{4}^{2}
$$

Isso nos fornece que o fator de segurança ao desgaste por crateramento no pinhão é de $1.33^{2}=1,77$ e $1,178^{2}=1,3877$. Ainda assim, observamos que em ambas engrenagens, a falha que está mais susceptível a acontecer é a por cratera, visto que os coeficientes de segurança de flexão são maiores.

Tabela 10: Tabela de componentes dimensionados

\begin{tabular}{|c|c|c|c|c|c|c|c|c|}
\hline \multicolumn{4}{|c|}{ CABO DE AÇO } & \multicolumn{3}{|c|}{ TAMBOR DO CABO DE AÇO } & \multicolumn{2}{|c|}{ EIXO CD } \\
\hline $\begin{array}{l}\text { Fator de } \\
\text { segurança }\end{array}$ & $\mathrm{CT}(\mathrm{kN})$ & $\begin{array}{l}\text { CRM } \\
(\mathrm{kN})\end{array}$ & $\mathrm{CRM}(\mathrm{tf})$ & $\begin{array}{l}\text { Diâmetro } \\
(\mathrm{mm})\end{array}$ & $\mathrm{Ma}(\mathrm{N} . \mathrm{m})$ & $\operatorname{Tm}(\mathrm{N} . \mathrm{m})$ & $\begin{array}{l}\text { Fator de } \\
\text { segurança }\end{array}$ & $\begin{array}{l}\text { Diâmetro } \\
\text { (mm) }\end{array}$ \\
\hline 8 & 7,325 & 58,6 & 5,977 & 315,5 & 527,94 & 1230,25 & 2 & 53,85 \\
\hline \multicolumn{9}{|c|}{ CORREIA } \\
\hline Tipo & Quantidade & Redução & $\begin{array}{c}\text { Diâmetro } \\
\text { polia motora } \\
\text { (mm) }\end{array}$ & $\begin{array}{c}\text { Diâmetro } \\
\text { polia movida } \\
(\mathrm{mm})\end{array}$ & $\begin{array}{c}\text { Circunferê } \\
\text { ncia } \\
\text { Interna Lc } \\
\text { (mm) }\end{array}$ & $\begin{array}{c}\text { Distânica } \\
\text { entre } \\
\text { centros C } \\
(\mathrm{mm})\end{array}$ & $\begin{array}{c}\text { Tempo de } \\
\text { vida (h) }\end{array}$ & $\begin{array}{l}\text { Fator de } \\
\text { segurança }\end{array}$ \\
\hline 8 & 2 & 5,46 & 155 & 846,3 & 4500 & 1445 & 87000 & 1,45 \\
\hline \multicolumn{9}{|c|}{ CORRENTE } \\
\hline $\begin{array}{c}\text { Configuraç } \\
\text { ão }\end{array}$ & Redução & Hproj (cv) & Heorrig (cv) & $\begin{array}{l}\text { Capacidade } \\
\text { de corrente } \mathrm{n}^{\circ}\end{array}$ & $\begin{array}{c}\text { Diâmetro } \\
\text { pinhão } \\
\text { (in) }\end{array}$ & $\begin{array}{l}\text { Diâmetro } \\
\text { coroa (in) }\end{array}$ & Número & de passos \\
\hline Simples & 4 & 5,04 & 3,93 & 60 & 3,37 & 13,37 & & 96 \\
\hline \multicolumn{9}{|c|}{ ENGRENAGENS } \\
\hline $\begin{array}{l}\text { Módulo m } \\
\text { (mm) }\end{array}$ & $\begin{array}{l}\text { Número de } \\
\text { dentes } \\
\text { pinhão }\end{array}$ & $\begin{array}{c}\text { Diametro } \\
\text { primitivo } \\
\text { pinhão } \\
(\mathrm{mm})\end{array}$ & $\begin{array}{l}\text { Número de } \\
\text { dentes coroa }\end{array}$ & $\begin{array}{l}\text { Diametro } \\
\text { primitivo } \\
\text { coroa (mm) }\end{array}$ & $\begin{array}{c}\text { Fator de } \\
\text { segurança } \\
\text { contra } \\
\text { flexão } \\
\text { pinhão } \\
\end{array}$ & \begin{tabular}{|c} 
Fator de \\
segurança \\
contra \\
flexão \\
coroa
\end{tabular} & $\begin{array}{c}\text { Fator de } \\
\text { segurança } \\
\text { contra } \\
\text { desgaste } \\
\text { pinhão } \\
\end{array}$ & $\begin{array}{c}\text { Fator de } \\
\text { segurança } \\
\text { contra } \\
\text { desgaste } \\
\text { coroa }\end{array}$ \\
\hline 6 & 18 & 108 & 90 & 540 & 3,75 & 4,53 & 1,33 & 1,178 \\
\hline
\end{tabular}

Fonte: Própria (2019)

E Segue abaixo na tabela (11) o balanço orçamentário dos componentes mecânicos mais relevantes estudados nessa produção: 
Tabela 11: Levantamento orçamentário do projeto do sistema de transmissão

\begin{tabular}{|c|c|}
\hline \multicolumn{2}{|c|}{ Orçamentos } \\
\hline THEM & PRECO \\
\hline Motor elétrico 4,0 cv 1800 rpm & RS $1.466,00$ \\
\hline Cabo de aço polido $9.53 \mathrm{~mm}$ & RS 4.449,00 \\
\hline Correia industrial em V B177 & RS 47,00 \\
\hline Corrente de tranmissilo & RS 295,00 \\
\hline Roda dentada 14 dentes & RS 37.00 \\
\hline Roda dentada 56 dentes & RS 435,00 \\
\hline TOTAL: & RS 6.294,00 \\
\hline
\end{tabular}

Fonte: Própria (2019)

\section{CONCLUSÕES}

Realizar esse trabalho permite não somente aplicar os conceitos de seleção de materiais e dimensionamentos de elementos de máquinas como também integrar nossa visão no que diz respeito ao funcionamento de conjuntos mecânicos. Tomar a liberdade de projetar, fazer considerações a respeito de aspectos construtivos e aplicar metodologias de grande respaldo para a obtenção dos resultados.

É um trabalho multidisciplinar no âmbito da engenharia mecânica e nos permite deixar uma contribuição para o nosso campus, não só na pesquisa e produção acadêmica, como também para o laboratório de práticas mecânicas que possui um norte para a futura instalação do sistema de içamento.

\section{REFERÊNCIAS}

BIANCHI, Kleber Eduardo. Desenvolvimento de um sistema de transmissão mecânica baseado em engrenagens cônicas e faciais. Disponível em: < https://www.lume.ufrgs.br/handle/10183/17877 > Acesso em 28 de julho de 2019;

COLlins, J. A., Projeto Mecânico de Elementos de Máquinas - Uma Perspectiva de Prevenção de Falha, LTC, Rio de Janeiro, 2006;

FARIA, Iolanda Balcky. Seleção de um redutor de engrenagens para um agitador e planejamento das ações de manutenção. Disponível em: 
<https://run.unl.pt/bitstream/10362/2511/1/Faria_2009.pdf $>$ Acesso em 29 de julho de 2019;

Manual técnico de cabos de Aço, Cimaf, agosto de 2012;

MELCONIAN, Sarkis. Mecânica Técnica e Resistência dos Materiais. 17 ed., Erica, 2006.

NIEMANN, G. Elementos de Máquinas. 6. ed. São Paulo: Edgard Blucher, 2002, vol. 2;

NORTON, R., Projeto de máquinas, Bookman, Porto Alegre, 2004;

NBR 4309, Equipamentos de movimentação de carga - Cabos de Aço - Cuidados, manutenção, instalação, inspeção e descarte;

SANTOS JUNIOR, A. A. dos. Engrenagens Cilíndricas de Dentes Retos. Campinas, 2002. Disponível em: < http://www.fem.unicamp.br/ lafer/em618/pdf/Apostila\%20Engrenagens\%2 >. Acesso em: 29 julho. 2019;

SHIGLEY, J. E., Elementos de Máquinas, Vol. 8, 8ed., LTC, Rio de Janeiro, 2008. 